\title{
ALTERAÇÕES SIALOQUÍMICAS E SIALOMÉTRICAS DE PACIENTES COM PARALISIA CEREBRAL: UMA REVISÃO DE LITERATURA
}

\section{Sialochemical and sialometric alterations in patients with cerebral palsy: a literature review}

\author{
Miriam Yumi Matsui (1), Maria Julia Pereira Coelho Ferraz ${ }^{(2)}$, \\ Mônica Fernandes Gomes ${ }^{(3)}$, Cybelle Mori Hiraoka ${ }^{(4)}$
}

\begin{abstract}
RESUMO
Tema: paralisia cerebral e alterações salivares. O paciente com paralisia cerebral é acometido por diversas desordens no Sistema Estomatognático, sendo muitas delas expressas sob a forma de alterações no fluxo e composição salivar. A variação da concentração de constituintes da saliva está diretamente relacionada com sua capacidade tampão, antioxidante, imunológica, digestiva e lubrificante, além de sofrer variações em função da velocidade do fluxo salivar, o qual está intimamente relacionado à eficiência dos estímulos mecânicos e neurais do trato salivar. Alterações na deglutição, da percepção gustativa, do processo de mineralização dos dentes e da propriedade protetora da saliva contra lesões cariosas, infecções e inflamações, freqüentemente observadas em pacientes com paralisia cerebral, podem ser avaliadas pelo exame da saliva. Objetivo: realizar uma revisão de literatura relacionando as principais alterações sialométrica e sialoquímica de pacientes com paralisia cerebral e seus efeitos na saúde bucal. Conclusão: a análise sialométrica e sialoquímica oferece informações extremamente úteis no diagnóstico e no direcionamento do tratamento desses pacientes, e pode ser considerada uma indicadora prática e objetiva dos processos de doença e disfunções.
\end{abstract}

DESCRITORES: Saliva; Bioquímica; Paralisia Cerebral

\section{INTRODUÇÃO}

O paciente com paralisia cerebral, em diversos níveis, enfrenta uma variedade de dificuldades: limitações funcionais motoras e cognitivas, dor crônica, perda de sensibilidade e acuidade visual, problemas de mastigação, fonação e gastrointestinais ${ }^{1}$.

(1) Cirurgiã Dentista; aluna de Mestrado em Biopatologia Bucal, Faculdade de Odontologia de São José dos Campos, UNESP, São Paulo, Brasil.

(2) Cirurgiã Dentista; Pós-Doutoranda em Biopatologia Bucal, Faculdade de Odontologia de São José dos Campos, UNESP, São Paulo, Brasil.

(3) Cirurgiã Dentista; Professora Adjunta da Disciplina de Patologia Bucal do Departamento de Biociências e Diagnóstico Bucal, Faculdade de Odontologia de São José dos Campos, UNESP, São Paulo, Brasil.

(4) Cirurgiã Dentista; Doutoranda em Biopatologia Bucal, Faculdade de Odontologia de São José dos Campos, UNESP, São Paulo, Brasil.

Conflito de interesses: inexistente
O tratamento requer uma abordagem de diversas áreas relacionadas à saúde, sendo que a Odontologia contribui no diagnóstico e na reabilitação oral desses pacientes.

É nesse contexto que a análise de alterações no fluxo e composição da saliva possui grande importância, já que a saliva contém inúmeras informações relacionadas aos estados emocional, hormonal, imunológico, neurológico, nutricional e metabólico ${ }^{2}$ favorecendo o direcionamento do tratamento desses pacientes.

Este trabalho objetiva, por meio de revisão de literatura, discorrer sobre as alterações sialométricas e sialoquímicas, assim como seus efeitos na saúde de pacientes com paralisia cerebral.

\section{MÉTODOS}

Foi realizada uma pesquisa na literatura, em bases de dados eletrônicas (PubMed, SciELO, e 
LILACS), a respeito da característica da saliva de pacientes com paralisia cerebral, incluindo artigos publicados em periódicos da literatura nacional e internacional e livros, desde as 3 últimas décadas (1979 a 2010), sendo $72 \%$ deles apresentados nos últimos 10 anos. Dissertações, teses, trabalhos de conclusão de curso, anais de congressos, documentos eletrônicos e materiais não publicados não foram considerados nesta pesquisa.

\section{REVISÃO DE LITERATURA}

\section{Paralisia cerebral}

A paralisia cerebral, também denominada encefalopatia crônica não-progressiva da infância, advém de lesão cerebral primária, podendo ser provocada pela falta de oxigenação das células cerebrais no período pré, peri ou pós-natal, afetando o sistema nervoso central em fase de maturação estrutural e funcional. Como conseqüência, resulta em déficits posturais, tônicos e na execução dos movimentos ${ }^{3-5}$.

Estas desordens do desenvolvimento motor ocasionam alterações músculo-esqueléticas, que por sua vez implicam em limitações das atividades físicas e saúde geral do indivíduo ${ }^{6}$.

As seqüelas são variáveis em intensidade e localização, dependendo da área do encéfalo que foi afetada, bem como da extensão da lesão. Não há dois casos de paralisia cerebral exatamente iguais. Mesmo nos casos mais leves, o comprometimento motor, embora sendo mínimo, afeta o bem estar psicológico destes indivíduos, gerando ansiedade e reforço de padrões estereotipados de postura ${ }^{7}$.

O envolvimento das áreas motoras do cérebro limita o desenvolvimento motor dos órgãos fonoarticulatórios, com persistência patológica de reflexos que impede a utilização adequada destes órgãos na alimentação e desenvolvimento de atividades musculares mais elaboradas ${ }^{8}$.

Como conseqüência, freqüentemente ocorrem complicações gerais e bucais em pessoas com paralisia cerebral. Uma alta prevalência de doenças orais são características nesses pacientes 9-11, como más oclusões, mordidas abertas anteriores, aumento no comprimento do arco superior, palato ogival, respiração bucal, bruxismo e presença de resíduos alimentares, algumas condicionadas pelo fator agravante da disfunção muscular ${ }^{12,13}$

Uma vez que a dificuldade no vedamento labial e a perda dos reflexos orais dificultam a dinâmica orofaríngea, são também comumente relatados: dificuldade na deglutição, escoamento externo da saliva, disfagia e persistência de reflexos primitivos orais, com incapacidade de controlar o alimento na boca, aspiração de saliva ${ }^{14}$, além de infecções pulmonares e das vias aéreas superiores ${ }^{15,16}$.

A deficiência de estimulação mecânica e a conseqüente diminuição dos estímulos neurais, por sua vez resultam na disfunção do trato salivar ${ }^{17}$. É de se esperar, dessa maneira, que pacientes com paralisia cerebral apresentem alterações sialoquímicas e sialométricas.

\section{Alterações sialoquímicas e sialométricas de pacientes com Paralisia Cerebral}

A prevalência de escape de saliva em pacientes com paralisia cerebral tem sido estimada entre $12 \%$ e $58 \%{ }^{18}$.

Essa alta taxa de escape oral, medida segundo a escala Teacher Drooling ${ }^{19}$ (escala de 1 a 5 sendo que 1 representa ausência e 5 o maior valor de escape oral de saliva, respectivamente) pode ser considerada alta (valor $=3)^{20}$, sendo que a deglutição e a mastigação apresentam alterações, sendo por vezes necessário tratamento com mucolíticos devido a freqüência da tosse decorrente do acúmulo de saliva espessa na garganta.

É importante salientar que o escape de saliva não é decorrente de hipersalivação. Por meio da análise comparativa do fluxo salivar em pacientes com paralisia cerebral e em pacientes saudáveis, pode ser verificado que não há diferenças significativas entre os grupos e que portanto, o escape ocorre decorrente da deficiência de controle motor $^{21}$.

Existem trabalhos, inclusive, em que a média de fluxo salivar para o grupo com paralisia cerebral é até menor $\left(0.26 .10^{-8}\right.$ metros cúbicos por segundo $\left.\left(\mathrm{m}^{3} / \mathrm{s}\right)\right)$ que os valores do grupo controle obtidos $\left(1.05 .10^{-8} \mathrm{~m}^{3} / \mathrm{s}\right)$, podendo ser considerada como estatisticamente significante ${ }^{22,23}$.

Em relação à concentração dos componentes salivares, podem encontrados valores aumentados de potássio $\left(20 \pm 3.10^{3} \mathrm{~mol}\right.$ de carga por metro cúbico $\left.\left(\mathrm{mmol}_{\mathrm{c}} / \mathrm{m}^{3}\right)\right)$, cálcio $\left(2.7 \pm 0.9 .10^{3} \mathrm{mmol} / \mathrm{m}^{3}\right)$, H2PO4- $\left(8.0 \pm 3.2 .10^{3} \mathrm{mmol} / \mathrm{m}^{3}\right)$, uréia (porcentagem em massa de $14.7 \pm 5.2 .10^{-3}$ quilograma $(\mathrm{kg} \%))$ e proteínas totais $\left(317 \pm 168.10^{-2} \mathrm{~kg} / \mathrm{m}^{3}\right)$, em comparação a sujeitos controle (potássio = $16 \pm 4.10^{3} \mathrm{mmol} / \mathrm{c}^{3}$, cálcio $=2.1+0.3 .10^{3} \mathrm{mmol} / \mathrm{m}^{3}$, $\mathrm{H} 2 \mathrm{PO} 4=4 \cdot 2+0 \cdot 6 \cdot 10^{3} \mathrm{mmol} / \mathrm{c}^{3}$, uréia $=6 \cdot 6+2 \cdot 5 \cdot 10^{-3}$ $\mathrm{kg} \%$ ) e proteínas totais $205 \pm 78 \cdot 10^{-2} \mathrm{~kg} / \mathrm{m}^{3}$ ). Já em relação aos componentes sódio $\left(7+4.8 .10^{3}\right.$ $\left.\mathrm{mmol} / \mathrm{m}^{3}\right)$ e cloreto $\left(14 \pm 4.10^{3} \mathrm{mmol} / \mathrm{m}^{3}\right)$ valores reduzidos foram encontrados em sujeitos com paralisia cerebral, em comparação a sujeitos controle (sódio $=33 \pm 18.10^{3} \mathrm{mmol} / \mathrm{c}^{3}$, cloreto $=26 \pm 13.10^{3}$ $\left.\mathrm{mmol} / \mathrm{m}^{3}\right)^{22}$.

As alterações dos parâmetros analisados sugerem maior risco de doenças orais nos pacientes com paralisia cerebral ${ }^{22,23}$. 
Diante disso, fica evidente a importância do emprego da análise da saliva como recurso diagnóstico, tornando-se necessária a compreensão da característica da saliva normal como padrão de comparação com as alterações apresentadas na paralisia cerebral.

\section{Saliva como recurso diagnóstico}

Diversas doenças sistêmicas podem comprometer o funcionamento das glândulas salivares e conseqüentemente, a produção de saliva, interferindo tanto na sua quantidade quanto na qualidade. Essas mudanças podem servir de parâmetro para o diagnóstico de diversas alterações. A avaliação funcional da saliva pode ser feita por métodos sialométricos (fluxo salivar) e sialoquímicos (a partir do exame da contagem de elementos orgânicos e inorgânicos). Este tem aplicação na identificação de agentes biológicos causadores de doenças virais, bacterianas e fúngicas. Além disso, pode ser indicador biológico importante no diagnóstico e prognóstico de neoplasias malignas, na medição de níveis hormonais, na avaliação do risco de cárie, dentre outros. Dentre as vantagens do uso desse recurso constam a facilidade de coleta e manuseio do material, além de baixo risco de contaminação para o operador ${ }^{24}$.

A saliva pode ser coletada diretamente de uma glândula (maior) ou recolhida do meio bucal para representar uma mistura das secreções das glândulas salivares, do fluído gengival, de células epiteliais descamadas, de restos de alimentos, de microorganismos e dos produtos de seu metabolismo ${ }^{25}$. A coleta da secreção de uma glândula isoladamente pode ser útil na avaliação da função dessa glândula em-específico. A coleta da saliva total é avaliada com o objetivo de elucidar alterações sistêmicas.

A coleta da saliva pode ser feita de forma estimulada ou não estimulada. A estimulação da produção de saliva, de forma mecânica (goma de mascar, parafina, látex) ou química (ácido cítrico), afeta a quantidade da saliva produzida. Portanto, alguns de seus constituintes também são alterados ${ }^{2}$. A coleta não estimulada é feita sem estímulos exógenos, entretanto o fluxo salivar também pode ser alterado por estímulos olfatórios, exposição à luminosidade, posição do corpo e ciclo circadiano ${ }^{25}$.

\section{Bioquímica da saliva}

A saliva, uma solução diluída e rica em substâncias orgânicas e inorgânicas, apresenta importante função na manutenção da integridade da mucosa oro-faringeana ${ }^{26}$.

Em pacientes saudáveis, existe uma variabilidade normal na concentração de substâncias salivares entre os indivíduos. Fatores variáveis como nutrição, características fisiológicas na saliva específicas e polimorfismo genético podem promover modificações no conteúdo e na função da saliva ${ }^{27,28}$.

Alguns componentes orgânicos atuam como fatores antimicrobianos (lactoferin, lisozima, lactoperoxidase, deferins), enquanto, outros são protetores de mucosa (glicoproteínas, imunoglobulina A (IgA) secretora, fator de crescimento epidermal, fator de crescimento fibroblástico básico). Estes aceleram, significativamente, o processo de reparação de feridas cruentas da mucosa gástrica e da cavidade bucal ${ }^{29,30}$. Além disso, contribuem para a lubrificação do bolo alimentar, favorecendo sua deglutição ${ }^{31}$.

Em relação às substâncias inorgânicas, o cálcio e o fósforo atuam diretamente no mecanismo de calcificação tecidual, modulando a desmineralização e remineralização. A capacidade tampão da saliva e seu $\mathrm{pH}$ são modulados por produtos de nitrogênio, como amônia e uréia, bicarbonatos e fosfatos. $\mathrm{O}$ pH normal da saliva é entre 6 e 7, significando que ela é ligeiramente ácida ${ }^{32}$.

Além dos estados metabólicos e imunológicos, a saliva reflete nos estados emocional, hormonal, neurológico e nutricional ${ }^{2}$.

A dosagem de cortisol na saliva proporciona um método fidedigno para avaliar a função adrenal, além de alterações como ansiedade, hiperatividade e déficit de atenção ${ }^{33}$.

Fluxo salivar, $\mathrm{pH}$, osmolaridade e a maioria de componentes salivares foram significantemente correlacionados com ansiedade, consciência, astúcia e introversão ${ }^{34}$. Dessa maneira, uma alteração na qualidade e quantidade de saliva, além de permitir a detecção de problemas infecciosos, imunológicos e hormonais, pode ser ainda um indicador de alterações psicológicas que é de notório interesse para o tratamento de pacientes com paralisia cerebral.

Uma vez discutidas as características da saliva, pode-se compreender a relevância do seu estudo, bem como as conseqüências de suas alterações no paciente com paralisia cerebral.

\section{DISCUSSÃO}

Impactos das alterações na composição e fluxo salivar em pacientes com paralisia cerebral

Apesar dos diversos estudos sobre o escape de saliva da cavidade oral, são poucas as pesquisas que analisam suas alterações bioquímicas. A tabela 1 destaca as diferenças na sialometria e sialoquímica de pacientes com paralisia cerebral em comparação a pacientes saudáveis encontradas em alguns estudos ${ }^{22,23}$. 
Tabela 1 - Comparação da sialometria e sialoquímica de pacientes com e sem paralisia cerebral

\begin{tabular}{|c|c|c|}
\hline \multirow{2}{*}{ Componentes salivares } & \multicolumn{2}{|c|}{ Valores encontrados na literatura ${ }^{22,23}$} \\
\hline & Maior que o grupo Controle & Menor que o grupo controle \\
\hline Fluxo salivar & & $x^{22,23}$ \\
\hline Proteínas totais & $X^{22,23}$ & \\
\hline Amilase & & $x^{23}$ \\
\hline Peroxidase & & $x^{23}$ \\
\hline Ácido siálico & $x^{22}$ & \\
\hline Sódio & & $x^{22}$ \\
\hline Potássio & $x^{22}$ & \\
\hline Cálcio & $x^{22}$ & \\
\hline Cloreto & & $x^{22}$ \\
\hline Uréia & $x^{22}$ & \\
\hline
\end{tabular}

Fonte: Davis ${ }^{22}$, New York, USA (1979) e Rodrigues Santos et al ${ }^{23}$, São Paulo, Brasil (2007)

Apesar do maior escoamento de saliva externamente à cavidade oral, os pacientes com paralisia cerebral apresentam redução no fluxo salivar (Tabela 1). Nos pacientes em que o tratamento empregado para o controle do escape é cirúrgico, a redução do fluxo é ainda maior. Esta redução de fluxo salivar no paciente com paralisia cerebral compromete a capacidade tampão e a quantidade absoluta de constituintes antibacterianos da saliva, indicando maiores riscos à lesões cariosas e inflamatória ${ }^{35}$.

A variação da viscosidade indica alterações na constituição salivar, particularmente, devido ao excesso de secreção de glicoproteínas salivares ${ }^{36}$. Apesar da função protetora e lubrificante das proteínas salivares, seu acúmulo levando ao aumento da viscosidade é extremamente desconfortável, comprometendo as funções de mastigação, fonação e deglutição, além do risco de rejeição social ${ }^{37}$.

A saliva possui um papel importante na fase inicial de digestão, pela ação da amilase salivar e ptialina ${ }^{38}$. Sua redução sugere a redução dessa função.

Peroxidases são enzimas antioxidantes, que previnem a formação de radicais livres. As peroxidases exercem função antibacteriana, catalizando metabólicos bacterianos e protegendo a mucosa dos efeitos de oxidação. A detecção de redução na peroxidase salivar sugere redução da eficiência enzimática antioxidante, podendo comprometer o equilíbrio do meio bucal ${ }^{39}$.

Nos processos biológicos, o acido siálico é mediador na adesão célula-célula, na comunicação intercelular, na renovação celular, nos receptores para bactérias e vírus ${ }^{40}$. Em pacientes com paralisia cerebral, a constatação de aumento pode estar relacionada à presença de infecções e inflamações no meio bucal.
As alterações nos níveis de sódio, cloreto, potássio, dentre outros, devem ser relacionados com a manutenção da isotonicidade da saliva, que permite a percepção gustativa da língua. Já a alteração de cálcio e fósforo podem alterar o equilíbrio de desmineralização e remineralização.

Por fim, a uréia possui papel na capacidade tampão da saliva e seu aumento pode sugerir prejuízos na neutralização da acidez salivar. Entretanto, a capacidade tampão é modulada também por outras substâncias, como amônia, bicarbonatos e fosfatos, além do fluxo salivar ${ }^{27}$.

Diante do exposto, pode-se observar que diversas alterações no fluxo e composição da saliva podem ser identificadas em pacientes com paralisia cerebral, e que muitas delas expressam o aumento de riscos à saúde bucal.

A análise do fluxo e composição da saliva é fundamental para que o cirurgião dentista compreenda o meio intra-bucal desses pacientes no intuito de atuar em sua reabilitação com cuidados direcionados. Seu emprego nos controles de tratamentos reabilitadores, bem como em pesquisas comparativas da saúde bucal pré e pós-tratamento de pacientes com paralisa cerebral também é viável e explica sua importância.

\section{CONCLUSÃO}

Os pacientes com paralisia cerebral apresentam limitações físicas e motoras com repercussões na biomecânica mandibular, com conseqüente disfunção do trato salivar.

A análise do fluxo e variações bioquímicas da composição salivar pode expressar a piora da condição bucal com reflexos práticos no diagnóstico e tratamento das disfunções do sistema estomatognático. 


\begin{abstract}
Background: cerebral palsy and salivary alterations. Patients with cerebral palsy frequently suffer from several disorders in the Stomatognathic System, many of them being expressed as alterations in the salivary flow and composition. Variations in the concentration of salivary components are directly related to their buffering, antioxidant, immunological, digestive and lubricant capacity, and vary according to the velocity of salivary flow, which is deeply related to the efficiency mechanical and neural stimuli on salivary tracts. Alterations in the swallowing function, gustative perception, mineralization of teeth process, as well as salivary protection function against caries lesions, infection and inflammation, which are frequently found in patients with cerebral palsy, can be analyzed through salivary exams. Purpose: this study aimed to perform a literature review related to the main sialometric and sialochemical alterations observed in patients with cerebral palsy and their effects on oral health. Conclusion: sialometric and sialochemical analyses are an objective and practical indicator of disease and dysfunction process and offer extremely useful information on the diagnosis and treatment referral for these patients.
\end{abstract}

KEYWORDS: Saliva; Biochemistry; Cerebral Palsy

\section{REFERÊNCIAS}

1. Odding E, Roebroeck ME, Stam HJ. The epidemiology of cerebral palsy: incidence, impairments and risk factors. Disabil Rehabil. 2006; 28(4): 183-91.

2. Mandel ID. The diagnostic uses of saliva. J Oral Pathol Med. 1990; 19(3): 119-25.

3. Castro CC, Batistela F, Martini G, Fonseca J, Montesanti L, Oliveira MC. Correlação da função motora e o desempenho funcional nas atividades de auto-cuidado em grupo de crianças portadoras de paralisia cerebral. Med Reabil. 2006; 25(1): 7-11. 4. Koman LA, Smith BP, Shilt JS. Cerebral palsy. Lancet. 2004; 363(9421): 1619-31.

5. Mancini MC, Alves ACM, Schaper C, Figueredo EM, Sampaio RF, Coelho ZA, et al. Gravidade da paralisia cerebral e desempenho funcional. Rev Bras Fisioter. 2004; 8(3): 253-60.

6. Molin I, Alricsson M. Physical activity and health among adolescents with cerebral palsy in Sweden. Int J Adolesc Med Health. 2009; 21(4): 623-33.

7. Nunes LCBG, Quevedo AAF, Magdalon EC. Effects of neuromuscular electrical stimulation on tibialis anterior muscle of spastic hemiparetic children. Rev Bras Fisioter. 2008; 12(4): 317-23.

8. Bell KL, Boyd RN, Tweedy SM, Weir KA, Stevenson RD, Davies PS. A prospective, longitudinal study of growth, nutrition and sedentary behaviour in young children with cerebral palsy. BMC Public Health. 2010; 10(1): 179.

9. Nunn JH, Murray JJ. The dental health of handicapped children in Newcastle and Northumberland. Br Dent J. 1987; 162(1): 9-14.
10. Pope J, Curzon ME. The dental status of cerebral palsied children. Ped Dent. 1991; 13(3): 156-62.

11. Dos Santos MT, Masiero D, Simionato MRL. Risk factors for dental caries in children with cerebral palsy. Spec Care Dentist. 2002; 22(3): 103-7.

12. Dos Santos MT, Nogueira ML. Infantile reflexes and their effects on dental caries and oral hygiene in cerebral palsy individuals. J Oral Rehabil. 2005; 32(12): 880-5.

13. Manzano FS, Granero LM, Masiero D, Dos Maria TB. Treatment of muscle spasticity in patients with cerebral palsy using BTX-A: a pilot study. Spec Care Dentist. 2004; 24(4): 235-9.

14. Bader CA, Niemann G. Dysphagia in children with cerebral palsy-fiberoptic-endoscopic findings. Laryngorhinootologie. 2010; 89(2): 90-4.

15. Troughton KEV, Hill AE. Relation between objectively measured feeding competence and nutrition in children with cerebral palsy. Dev Med Child Neurol. 2001; 43(3): 183-90.

16. Furkim AM, Behlau MS, Weckx LLM. Avaliação clínica e videofluoroscópica da deglutição em crianças com paralisia cerebral tetraparética espástica. Arq Neuro-Psiquiatr. 2003; 61(3A): 611-6.

17. Cheida AP. Hiperbolóide - Instrumento de Mastigação - um estimulador periomiofuncional. 1st ed. São Paulo: Ícone; 2004.

18. Tahmassebi JF, Curzon MEJ. Prevalence of drooling among children with cerebral palsy attending special schools. Dev Med Child Neurol. 2003; 45(9): 613-7.

19. Thomas-Stonell N, Greenberg J. Three treatment approaches and clinical factors in the reduction of drooling. Dysphagia. 1988; 3(2): 73-8. 
20. Erasmus CE, Van Hulst K, Van Den Hoogen FJ, Van Limbeek J, Roeleveld N, Veerman EC et al. Thickened saliva after effective management of drooling with botulinum toxin A. Dev Med Child Neurol. 2010; 52(6): 114-8.

21. Erasmus CE, Van Hulst $\mathrm{K}$, Rotteveel LJ, Jongerius PH, Van Den Hoogen FJ, Roeleveld N et al. Drooling in cerebral palsy: hypersalivation or dysfunctional oral motor control? Dev Med Child Neurol. 2009; 51(6): 454-9.

22. Davis MJ. Parotid Salivary Secretion and Composition in Cerebral Palsy. J Dent Res. 1979; 58(8):1808.

23. Rodrigues Santos MT, Siqueira WL, Nicolau J. Amylase and peroxidase activities and sialic acid concentration in saliva of adolescents with cerebral palsy. Quintessence Int. 2007; 38(6): 467-72.

24. Moura SAB, Medeiros AMC, Costa FRH, Moraes $\mathrm{PH}$, Oliveira Filho AS. Valor Diagnóstico da Saliva em Doenças Orais e Sistêmicas: Uma Revisão de Literatura. Pesq Bras Odontoped Clin Integr. 2007; 7(2): 187-94.

25. Kaufman E, Lamster IB. Analysis of saliva for periodontal diagnosis - a review. J Clin Periodontol. 2000; 27(7): 453-65.

26. Pedersen AM, Bardow A, Jensen SB, Nauntofte B. Saliva and gastrointestinal functions of taste, mastication, swallowing and digestion. Oral Dis. 2002; 8(3): 117-29.

27. Humphrey SP, Williamson RT. A review of saliva: Normal composition, flow, and function. J Prosthet Dent. 2001; 85(2): 162-9.

28. Aguirre A, Testa-Weintraub LA, Banderas JA, Haraszthy GG, Reddy MS, Levine MJ. Sialochemistry: a diagnostic tool? Crit Rev Oral Biol Med. 1993; 4(3-4): 343-50.

29. Epstein JB, Tsang AHF, Warkentin D, Ship $\mathrm{JA}$. The role of salivary function in modulating chemotherapy-induzed oropharyngeal mucosites: a review of the literature. Oral Surg Oral Med oral Pathol. 2002; 94(1): 39-44.

30. Magnusson M, Pyykkö I, Setten G, Norlander T, Nastri , Westermark A. Basic fibroblastic growth factor (bFGF) in saliva and oral mucosa in patient with oral lichen planus: preliminary observations. Oral Surg Oral Med Oral Pathol Oral Radiol Endod. 2004; 98(3): 324-6.

31. Prinz JF, Lucas PW. Swallow thresholds in human mastication. Arch Oral Biol. 1995; 40(5): 401-3.

32. Aframian DJ, Davidowitz T, Benoliel R. The distribution of oral mucosal $\mathrm{pH}$ values in healthy saliva secretors. Oral Dis. 2006; 12(4): 420-3.

33. Blomqvist M, Holmberg K, Lindblad F, Fernell E, Ek U, Dahllo"f G. Salivary cortisol levels and dental anxiety in children with attention deficit hyperactivity disorder. Eur J Oral Sci. 2007; 115(1): 1-6.

34. Costa PT Jr, Chauncey HH, Rose CL, Kapur KK. Relationship of parotid saliva flow rate and composition with personality traits in healthy men. Oral Surg Oral Med Oral Pathol. 1980; 50(5): 416-22.

35. Hallett KB, Lucas JO, Johnston T, Reddihough DS, Hall RK. Dental health of children with cerebral palsy following sialodochoplasty. Spec Care Dentist. 1995; 15(6): 234-8.

36. Waterman HA, Blom C, Holterman $\mathrm{HJ}$, 's-Gravenmade EJ, Mellema J. Rheological properties of human saliva. Arch Oral Biol, Oxford. 1988; 33(8): 589-96.

37. Meningaud JP, Pitak-Arnnop P, Chikhani L, Bertrand JC. Drooling of saliva: a review of the etiology and management options. Oral Surg Oral Med Oral Pathol Oral Radiol Endod. 2006; 101(1): 48-57.

38. Sivakumar T, Hand AR, Mednieks M. Secretory proteins in the saliva of children. J Oral Sci. 2009; 51(4): 573-80.

39. Ashby MT. Inorganic chemistry of defensive peroxidases in the human oral cavity. J Dent Res. 2008; 87(10): 900-14.

40. Fátima A, Baptistella LHB, Pilli A, Modolo LV. Ácidos siálicos - da compreensão do seu envolvimento em processos biológicos ao desenvolvimento de fármacos contra 0 agente etiológico da gripe. Quím Nova. 2005; 28(2): 306-16.

RECEBIDO EM: 05/08/2010

ACEITO EM: 01/09/2010

Endereço para correspondência:

Miriam Yumi Matsui

R. Laís Toledo Dantas de Matos, 230 -

Jardim Alzira - São Paulo - SP

CEP: 04655-080

E-mail: miriam.matsui@gmail.com 